\title{
Report von COMARE widerspricht deutscher Kinderkrebsstudie in der Umgebung von Kernkraftwerken
}

\author{
Britische Forscher haben die deutsche KiKK-Studie untersucht und erhebliche metho- \\ dische Mängel nachgewiesen. Zudem sei die im Normalbetrieb abgegebene Strah- \\ lung aus Kernkraftwerken um mehr als das 1000-fache zu klein, um ein erhöhtes \\ Leukämierisiko bei Kleinkindern zu erklären.
}

Jürg Schädelin

\section{Für das Forum Medizin} und Energie (FME)

Dr. med, ehemaliger medizinischer Leiter der Abteilung Epidemiologie und Medikamentensicherheit bei Novartis. Mitautor der FME-Broschüre «Kinderleukämie und Kernkraftwerke - (K)Ein Grund zur Sorge?»
Korrespondenz:

Dr. med. Christian von Briel Forum Medizin und Energie Postfach CH-8040 Zürich Tel. 0435011850

kontakt@fme.ch www.fme.ch
Die Expertengruppe COMARE (Comittee on Medical Aspects of Radiation in the Environment) wurde als permanente Nachfolge für die Black-Comission durch die Health Protection Agency in Grossbritannien eingesetzt und publiziert seither regelmässig Berichte über die vermuteten Gesundheitsschäden durch Kernkraftwerke und Nuklearanlagen im Vereinigten Königreich. Ihren vorletzten, zwölften Report widmete sie allerdings der deutlich gestiegenen Strahlenbelastung durch die zunehmenden CT-Untersuchungen im Gesundheitswesen. Dies stellt ihrer Meinung nach ein wesentlich ernsteres Problem dar als die vieldiskutierten Emissionen von Kernkraftwerken.

Wenn nun der soeben publizierte vierzehnte Report als Aufarbeitung der deutschen KiKK-Studie (Kinderkrebs in der Umgebung von Kernkraftwerken) doch wieder die Kinderleukämien in der Umgebung von Nuklearanlagen behandelt, so entspricht dies dem erneuten Interesse durch die deutschen Befunde und der neuartigen Methodologie, die in der KiKKStudie angewendet wurde. Während bisher die
In der Einleitung zum vierzehnten Report liefert COMARE eine kritische Rückschau zu den bisherigen Untersuchungen, insbesondere der vieldiskutierten KiKK-Studie. Da eine verlässliche Messung der Strahlenexposition schon für die Beschäftigten in Kernkraftwerken problematisch ist und für Zivilpersonen in der Umgebung nicht praktikabel erscheint, bleibt nur der geographische Abstand als Risikoparameter. Eine Fallkontrollstudie bringt jedoch nur dann Vorteile, wenn die ausgewählten Personen eingehend nach weiteren möglichen Risikofaktoren befragt werden können. Dies war in der deutschen Studie nicht möglich. In England wurde daher die gesamte Population verglichen. Eine präzise Schätzung des jeweiligen Krankheitsrisikos wurde hier durch die Tatsache erleichtert, dass Gesundheitsstatistiken in kleinräumigen Postleitzahlbezirken vorliegen. An der KiKKStudie wurde weiterhin bemängelt, dass die bekannten sozio-ökonomischen Risikofaktoren nicht berücksichtigt wurden und dass die Zeitperiode, deren Daten die Vermutung eines erhöhten Risikos für Kin-

\section{Eine Fallkontrollstudie bringt nur dann Vorteile, wenn die ausgewählten Personen nach weiteren möglichen Risikofaktoren befragt werden können. Dies war in der deutschen Studie nicht möglich.}

Gesundheitsstatistiken in vorgegebenen Räumen um Kernkraftwerke mit dem Landesdurchschnitt oder selektierten Vergleichsregionen verglichen wurden, analysierten die Epidemiologen des deutschen Kinderkrebsregisters in ihrer Fallkontrollstudie die exakte Entfernung vom Kernkraftwerk als Parameter für die angenommene Strahlenbelastung. Dabei fanden sie eine signifikante Risikozunahme für Kinder unter 5 Jahren in der näheren Umgebung von 5 Kilometern mit einer Steigerung der Inzidenz auf fast das Doppelte. der unter 5 Jahren generierte, in der folgenden, konfirmatorischen Analyse erneut eingeschlossen wurde.

Entsprechend dem Risikomodell der KiKK-Studie analysierten die britischen Forscher alle Krankheitsfälle von akuter Leukämie oder Non-Hodgkin-Lymphom im Zeitraum von 1969 bis 2004 . Von insgesamt 8838 Erkrankungen traten 430 in einem Bezirk auf, der näher als 25 Kilometer bei einem der 13 Kernkraftwerke Grossbritanniens lag. Allerdings wurden Sellafield und Dounreay nicht eingeschlossen mit der Begründung, dass dort auch eine Wiederaufberei- 


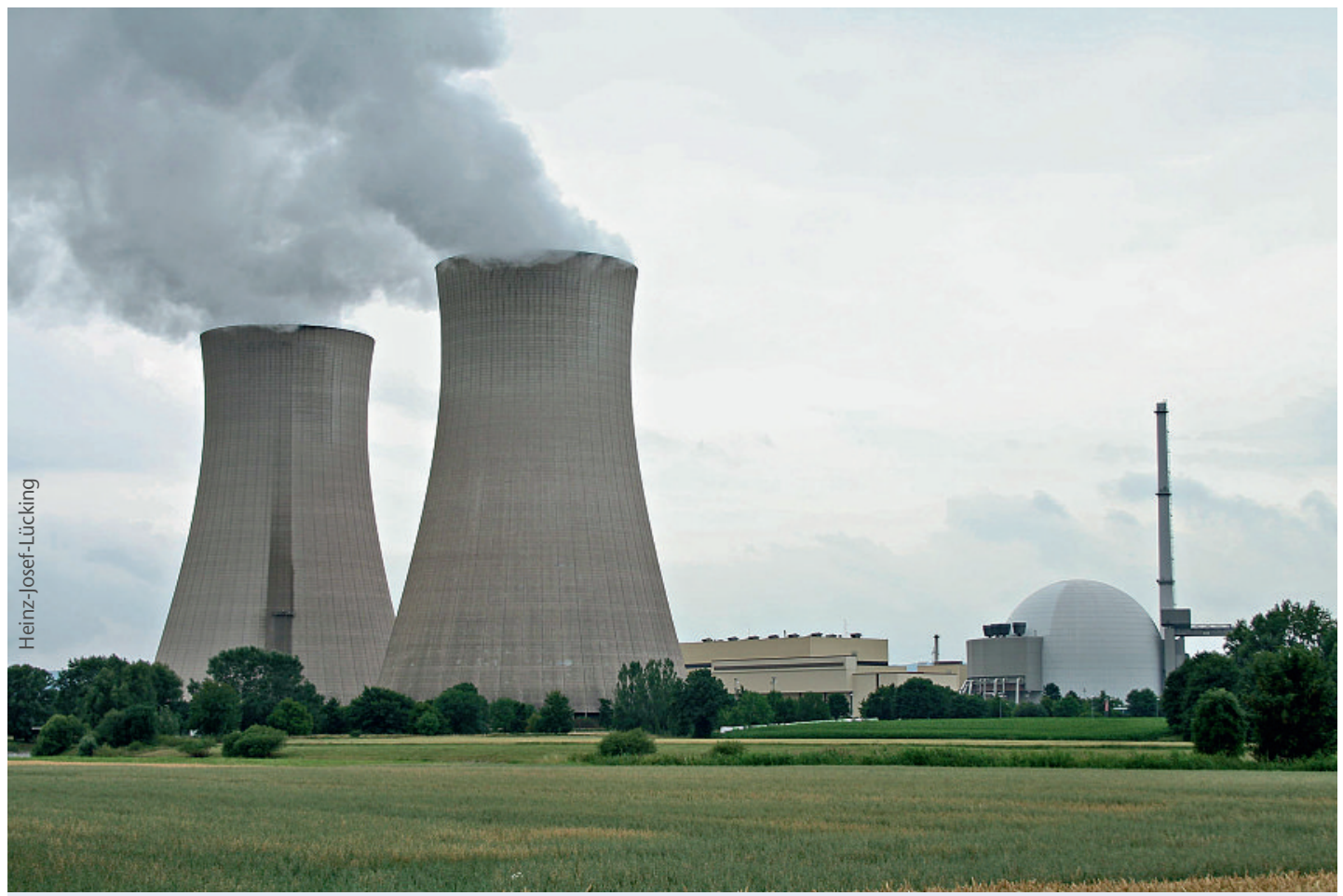

Ist erhöhtes Leukämie-Risiko bei Kindern auf das Wohnen in der Nähe eines Kernkraftwerks zurückzuführen?

tungsanlage beziehungsweise eine Produktionsstätte für Kernbrennstoffstäbe gelegen seien - Anlagen, die in Deutschland nicht vorkommen und deren erhöhtes Krankheitsrisiko die ursprüngliche Hypothese generierten. Obwohl dieses Vorgehen die Vergleichbarkeit mit der deutschen Analyse formal erleichtert, wäre eine separate Berechnung dieser Standorte nach der neuen Methode einer Kritik zuvorgekommen. Diese Berechnungen wurden für den nächsten Report versprochen.

Nach der logistischen Regressionsrechnung steigt das Leukämierisiko für das Reziproke des Abstands mit einer Steigung von $0,068 \pm 0,940$ pro Kilometer (95\% Konfidenzintervall $-1,77$ bis 1,91$)$. Eine Abstandsabhängigkeit ist also weit von einer statistischen Signifikanz entfernt. Für den Bereich unter 5 Kilometern um Kernkraftwerke wurden 20 Krankheitsfälle registriert, erwartet wurden nach Landesdurchschnitt 16,35 was einer Standard Incidence Ratio von 1,22 (CI 0,75-1,89) entspricht. In der Umgebung von 10 Kilometern fanden sich 61 Erkrankungen bei erwarteten 71,65 entsprechend einer SIR von 0,86 (CI 0,66-1,10).

Die gleiche Berechnung wurde auch für 6 potentielle Standorte durchgeführt, an denen ein Kernkraftwerk geplant, aber in der Folge nicht errichtet wurde. Dort findet sich ein signifikanter Anstieg des Risikos $(\beta=1,66 \pm 0,82)$ und im Umkreis von 5 Kilometern eine SIR von 1,72 (CI 1,12-2,52). Allerdings ist die Signifikanz ausschliesslich auf einen einzelnen
Bezirk zurückzuführen, und einige geplante Standorte liegen in der grossstädtischen Umgebung von London und sind nicht vergleichbar mit den bestehenden Lokalitäten für Kernkraftwerke.

Diese Studie beweist, dass die in Deutschland gefundene Erhöhung des Krankheitsrisikos in der näheren Umgebung von Kernkraftwerken im Normalbetrieb kein generelles Phänomen ist. Da die gemessenen radioaktiven Emissionen nach den heutigen Risikoabschätzungen für Strahlenbelastung um Grössenordnungen zu klein sind, um die wenigen - und meist passagären - Häufungen von Kinderleukämien zu erklären, müssen die Gründe in anderen Charakteristika dieser Standorte gesucht werden. Eine jüngst erschienene Übersicht von Kinlen [1] weist auf die regelmässig in der Folge von Bevölkerungsverschiebungen zu beobachtende Erhöhung der Leukämieinzidenz hin, die seit Jahrzehnten wiederholt festgestellt wurde. In vier von fünf gefundenen Leukämieclustern um Kernanlagen fanden sich derartige Bevölkerungsdurchmischungen in einem Ausmass, das die beobachteten Krankheitsraten zwanglos erklärt. Nur in Krümmel in Deutschland, das zur KiKKStudie den grössten Effekt beisteuert, wurde eine derartige Analyse bisher nicht vorgenommen. Es bleibt $\mathrm{zu}$ wünschen, dass die langjährigen und fundierten Abklärungen der COMARE-Gruppe die zum Teil emotional geführte Diskussion um dieses Thema auf den Boden der wissenschaftlichen Tatsachen zurückbringt. r J Cancer. 2011; 10 $12-8$. 